\title{
Molecular Mechanism of Xixin-Ganjiang Herb Pair Treating Chronic Obstructive Pulmonary Disease-Integrated Network Pharmacology and Molecular Docking
}

\author{
Ping Huang $\mathbb{D}^{1},{ }^{1}$ Tao Huang, ${ }^{2}$ Deshun Li, ${ }^{1}$ Lintao Han, ${ }^{3}$ Zhenxiang Zhou, ${ }^{1}$ Fang Huang, \\ Jingjing Li, ${ }^{1}$ Jiajia Wu, ${ }^{1}$ Yan Ye, ${ }^{3}$ Qiong Wang $\mathbb{D}^{1},{ }^{1}$ and Bailu Duan $\mathbb{D}^{1}$ \\ ${ }^{1}$ College of Basic Medicine, Hubei University of Chinese Medicine, Wuhan 430065, China \\ ${ }^{2}$ Wuhan Red Cross Hospital, Wuhan 430065, China \\ ${ }^{3}$ Pharmacy School, Hubei University of Chinese Medicine, Wuhan 430065, China
}

Correspondence should be addressed to Qiong Wang; wq_ccp@hbtcm.edu.cn and Bailu Duan; bailuduan2018@hbtcm.edu.cn

Received 15 January 2021; Revised 26 March 2021; Accepted 23 May 2021; Published 10 June 2021

Academic Editor: Dan Su

Copyright (C) 2021 Ping Huang et al. This is an open access article distributed under the Creative Commons Attribution License, which permits unrestricted use, distribution, and reproduction in any medium, provided the original work is properly cited.

Background. Chronic obstructive pulmonary disease (COPD) is characterized by high morbidity, disability, and mortality, which seriously threatens human life and health. Xixin and Ganjiang are classic herb pairs of Zhongjing Zhang, which are often used to treat COPD in China. However, the substance basis and mechanism of action of Xixin-Ganjiang herb pair (XGHP) in the treatment of COPD remain unclear. Methods. On the website of TCMSP and the DrugBank, effective compounds and targets of XGHP were found. COPD targets were obtained from GeneCards, DisGeNET, and GEO gene chips. Intersecting these databases resulted in a library of drug targets for COPD. Then, intersection targets were used for protein-protein interaction (PPI) and pathway enrichment analysis. Finally, the binding activity between compounds and core genes was evaluated by molecular docking to verify the expression level of PTGS2 and PPARG in rats. Results. Twelve effective compounds and 104 core genes were found in the intersection library, and kaempferol, sesamin, $\beta$-sitosterol, PTGS2, and PPARG were particularly prominent in the network analysis. A total of 113 pathways were obtained and enrichment of the TNF signaling pathway, IL-17 signaling pathway, and C-type lectin receptor signaling pathway was particularly obvious. Molecular docking indicated that kaempferol, sesamin, and $\beta$-sitosterol were closely related to PTGS2 and PPARG and were superior to aminophylline. Key compounds in XGHP could restrict the expression of PTGS2 in the lung tissues of COPD rats and promote the expression of PPARG. Conclusion. Inhibition of the expression of inflammatory factor PTGS2 and promotion of the expression of PPARG may be an effective target of XGHP in the treatment of COPD.

\section{Introduction}

Chronic obstructive pulmonary disease (COPD) is a progressive chronic respiratory disease with a high morbidity and mortality $[1,2]$. Airflow restriction, chronic bronchitis, and chronic airway obstruction may decline pulmonary function in a progressive and irreversible manner. Data published by the World Health Organization (WHO) showed that nearly 3 million people worldwide die from COPD each year [3]. Smoking, environmental effects, and occupational exposure are the main causes of COPD. The pathological changes of COPD are mainly immune reactions occurring in the central airway, small airway, and the alveolar space. The protease-antiprotease hypothesis, immune mechanism, oxidation-antioxidant balance, and systemic inflammation have all been considered to be related to the pathogenesis of COPD [4]. Takeuchi et al. suggested that the development of emphysema was related to these types of immune inflammation, which increase the production of mucus and disrupt the lungs' gas-exchange surfaces [5]. Moreover, chronic inflammation is key in COPD development, and abnormal distribution of neutrophils, macrophages, and lymphocytes has been found in the small airways of patients with COPD $[6,7]$. So far, no drugs for 
COPD have been proven to alter the long-term decline in pulmonary function, and bronchodilators are the primary treatment for COPD. By 2030, COPD is projected to be the third leading killer of human life and health [8]. The WHO has designated the Wednesday in the third week of November as World COPD Day to raise awareness and strengthen the prevention and treatment of COPD.

Historically, Traditional Chinese Medicine (TCM) has achieved a great curative effect in the treatment of various diseases, because of its integrated conditioning ability and few adverse reactions. In the process of treating diseases in TCM, different compatibility between herbs can play different therapeutic roles, even if medicine can contain dozens of herbs. Herb pair is the simplest compatibility, which contains only two herbs [9]. XGHP was derived from ShangHanZaBingLun, written by Zhongjing Zhang in the classic prescriptions of Xiaoqinglong decoction, Linggan Wuwei Jiangxin decoction, and other medications. XGHP is widely and effectively used to treat COPD. Xiaoqinglong decoction, which contains XGHP, has been shown to inhibit excessive airway mucus secretion, reduce airway obstruction, and improve lung ventilation function [10]. Linggan Wuwei Jiangxin decoction could regulate the content of MUC5AC in rats and regulate the secretion of airway fluid to treat lung disease with a Cold Syndrome $[11,12]$. However, the effective substance and underlying mechanism of action of the therapeutic effect of XGHP have not yet been identified, which restricts its clinical application and development.

Network pharmacology has been widely used to explore novel drugs and repurpose existing drugs [13]. Through the establishment of a "compound-gene-disease" network, it was more effective to reveal the regulation principle of small molecules with high throughput, compared with the contemporary "one target, one drug" mode [14]. Because of the complex composition in herbs and the multiple targets in diseases, network pharmacology has become an effective tool in predicting novel drug targets and mining the material basis of the TCM system.

In the current study, the core components and targets of XGHP were be predicted by network pharmacology and verified by molecular docking and in vivo verification to explore the active substances and pathways of the herb pair. The working flowchart is presented in Figure 1.

\section{Materials and Methods}

2.1. Collection of the Small Molecule of XGHP and Screening. In this study, we followed the methods of Duan et al. 2020 [15] to obtain chemical compounds in Xixin (XX) and Ganjiang (GJ) from TCMSP (http://tcmspw.com/tcmsp. php) [16]. Based on literature reports, ingredients that met $\mathrm{OB} \geq 30 \%$ and $\mathrm{DL} \geq 0.18$ were screened out as the main active ingredients of herbs [17]. In addition, compounds were supplemented by literature retrieval and data mining. Potential protein targets of XGHP main compounds were corrected through UniProt (https://www.uniprot.org/) databases.
2.2. Establishment of Database for Targets Associated with COPD. Microarray data of differentially expressed RNA from alveolar macrophages in normal and COPD groups were downloaded from the GEO database (https://www. ncbi.nlm.nih.gov/geo/), series: GSE130928, platforms: GPL570-55999. The Limma package in the Bioconductor platform (https://www.bioconductor.org/) and R3.6.3 software were used for chip analysis. Genes with $P<0.05$ and $\log 2(\mathrm{FC})<>1$ or $\log 2(\mathrm{FC})<-1$ were selected, and these genes were thought to be significantly differentially expressed associated with COPD. In addition, disease genes from the Human Gene Database (https://www.genecards. org) and DisGeNET (https://www.disgenet.org) databases were combined, duplicate disease targets were eliminated, and a COPD disease targets database was established.

2.3. Creation of XGHP-COPD Network Diagrams. Next, the VEEN tool (http://bioinformatics.psb.ugent.be/cgi-bin/liste/ Venn/calculate_venn.htpl) was used to intersect the effective drug targets and disease targets, and the core targets of XGHPCOPD were obtained. The core targets were then uploaded into STRING (https://stringdb.org/cgi/input.pl), which provided information on the interaction of proteins [18] to construct the protein-protein interaction (PPI) network. Next, we used Cytoscape 3.7.2 (https://www.cytoscape.org/) and its attachments CytoNCA inside the parameters of the Degree of Centricity (DC), Closeness Centrality (CC), and Betweenness Centrality (BC) to further filter out core targets [19].

2.4. Analyses of Enrichment Pathway. The ClusterProfile package [20] was downloaded from the Bioconductor website and applied to R 3.6.3 software to obtain Gene Ontology (GO) and Kyoto Encyclopedia of Genes and Genomes (KEGG) enrichment information of overlapping targets. Subsequently, related "histograms" and "bubble graphs" were established.

\section{Validation}

3.1. Molecular Docking. To further examine the predictive power of our previous network pharmacology, molecular docking techniques were used to determine the ability of the screened active ingredients to bind to these proteins. The PDB website (http://www1.rcsb.org/) was used to download the protein structure, while its $3 \mathrm{D}$ form was available on the PubChem website (https://pubchem.ncbi.nlm.nih.gov/). Furthermore, PyMol software removed water molecules and small molecule ligands, and the receptor and ligand were hydrogenated by AutoDock.

\subsection{Efficacy Evaluation of XGHP in a COPD Rat Model}

3.2.1. Reagents. Xixin and Ganjiang were purchased from the TCM Pharmacy of Wuhan Hospital of Traditional Chinese Medicine (Wuhan, China). According to the ratio of $1: 1$, the amount of crude drug per unit body weight of rats was obtained according to the amount of crude drug per unit body weight of human, enlarged by 10 times to weigh the restorative materials and prepare the Xixin-Ganjiang decoction [21]. 


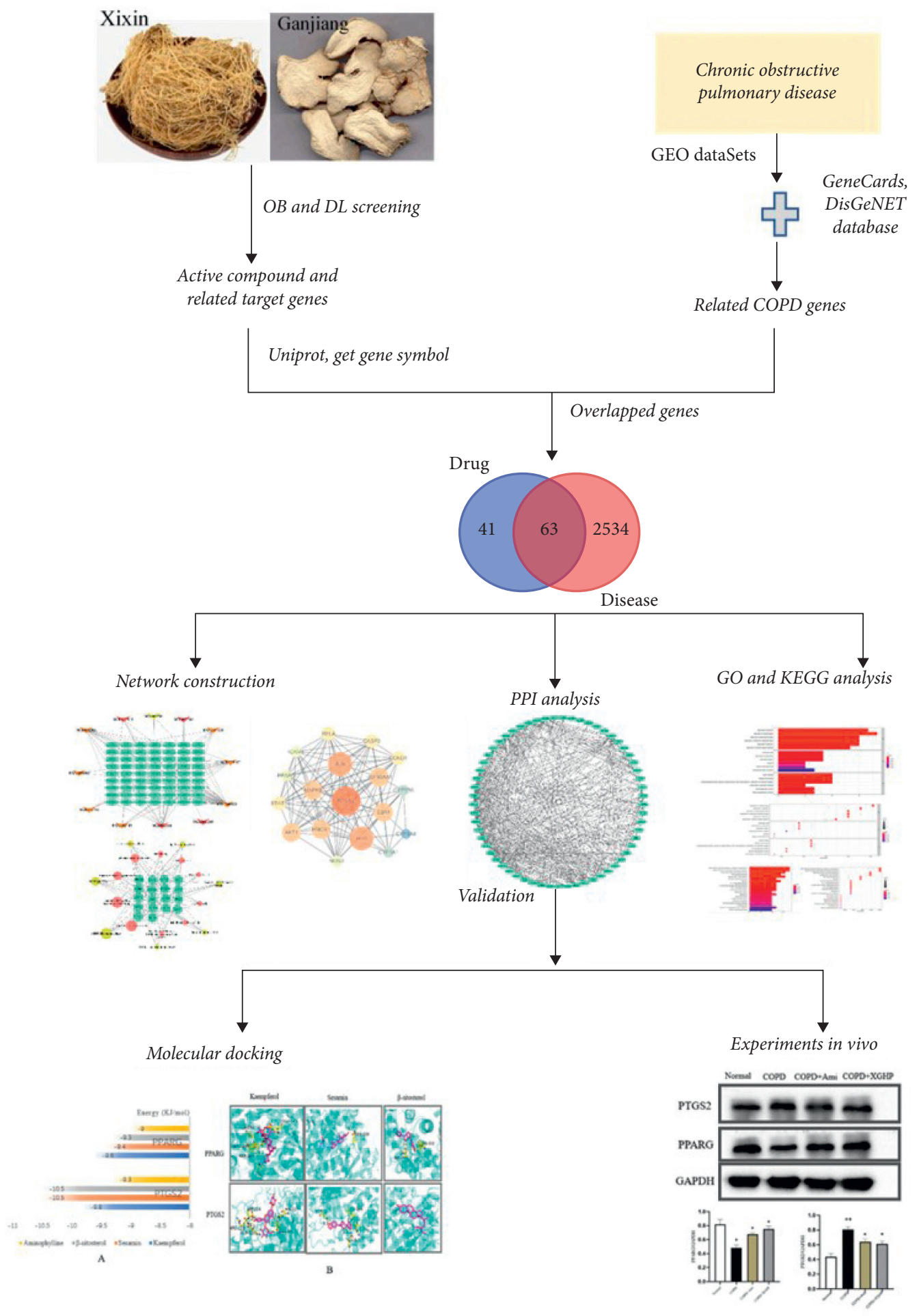

FIGURE 1: Flowchart of mechanism exploration of XGHP treatment for COPD.

Water was added to boil twice for $30 \mathrm{~min}$ and then filtered and concentrated at $60^{\circ} \mathrm{C}$ in a constant temperature water bath. Finally, the XGHP decocted concentration was $2.7 \mathrm{~g} /$ $\mathrm{ml}$, which was stored in the refrigerator for further use. The cigarettes were Yellow Crane Tower (Wuhan, China). Lipopolysaccharide was purchased from Sigma (St. Louis, MO, USA). Chloral hydrate was purchased from Tianjin
Damao Chemical Reagent Factory (Tianjin, China). Primary antibodies: anti-PTGS2(\#12282) was purchased from CST (Massachusetts, USA), anti-PPARG (GB11164), and anti-GAPDH (GB11002) were purchased from Servicebio (Wuhan, China). Secondary antibody: HRPlabeled Goat Anti-Rabbit lgG $(\mathrm{H}+\mathrm{L})$ was purchased from Servicebio (Wuhan, China). 
3.2.2. Animals. Adult Sprague Dawley (SD) male rats (220 250 g; aged 6-8 weeks; males) were recruited from Hubei Experimental Animal Research Center (Wuhan, China) and were fed in a specific pathogen-free (SPF) laboratory of Hubei University of TCM (Wuhan, China) at room temperature, a humidity of $50 \pm 10 \%$, and $12 \mathrm{~h}$ day and night light cycle. Rats were fed standard feed pellets and had free access to water. The Animal Ethics Committee of Hubei University of Chinese Medicine approved the study protocol.

3.2.3. Induction and Administration of COPD. Rats were randomly divided into four groups: normal group, COPD group, COPD + XGHP group, and a COPD + aminophylline (Ami) group (10 rats per group). Rats were injected with LPS ( $200 \mu \mathrm{l} /$ injection) on the first and 14th day, respectively. Rats, except for rats in the normal group, were exposed to 14 cigarettes of smoke for 30 minutes at 4-hour intervals in a smoke box from the second day to the 30th day (except for day 14 ), frozen at $0^{\circ} \mathrm{C}$ for $1 \mathrm{~h} / \mathrm{d}$, and given a mixture of ice and water by gacaged $(1 \mathrm{ml} / 100 \mathrm{~g})$ to replicate the model of cold drink accumulation of lung syndrome. Normal group rats were given control treatment. From day 16, rats in the normal group and COPD group were gavaged with $1 \mathrm{ml} / 100 \mathrm{~g}$ saline once a day, and rats in the COPD $+\mathrm{XGHP}$ group were gavaged with $1 \mathrm{ml} / 100 \mathrm{~g}$ traditional medicine decoction once a day. In the COPD + Ami group, $1 \mathrm{ml} / 100 \mathrm{~g}$ aminophylline solution $(5 \mathrm{~g} / \mathrm{l})$ was gavaged once a day for 14 days.

At the end of the experiment, lung tissues were removed under chloral hydrate anesthesia and labeled and stored in liquid nitrogen for Western blot analysis.

3.2.4. Western Blot Analysis. Lung tissues were weighed, minced with steel balls, and dissolved in $100 \mathrm{mmol} / \mathrm{L}$ PMSF radioimmunoprecipitation (RIPA) buffer. The homogenate was then centrifuged at $4^{\circ} \mathrm{C}$ and $12,000 \mathrm{rpm}$ for $10 \mathrm{~min}$. Then, the supernatant was collected and $5 *$ loading buffer was added proportionally. The solution was sterilized at $100^{\circ} \mathrm{C}$ heating water for $15 \mathrm{~min}$. Finally, it was cooled to room temperature and divided into Eppendorf tubes. The proteins were isolated and separated on a $12 \%$ SDS-PAGE and electrophoretically transferred onto PVDF membranes. Membranes were blocked with 5\% skim milk (TBST $-0.1 \%$ Tween-20, TBST) for 1 hour at room temperature and incubated with primary antibodies at $1: 1000$ dilution at $4^{\circ} \mathrm{C}$ overnight. Next, membranes were washed for 3 times with TBST solution ( $5 \mathrm{~min}$ for each), followed by incubation with HRP-labeled Goat Anti-Rabbit $\lg G(H+L)$ for $30 \mathrm{~min}$. Then, membranes were rinsed 3 times with TBST solution and the immunoreactive zone was observed with an enhanced chemiluminescence (ECL) reagent kit. The density of each band was analyzed by using Bio-Rad Quantity One software. GAPDH was selected as an internal reference for semiquantitative analysis.

3.2.5. Statistical Analysis. Statistical analysis and mapping were performed using GraphPad Prism 8 software (San Diego, CA, USA). Differences between groups were statistically compared by unpaired $t$-test. One-way analysis of variance was used for multiple comparisons. $P<0.05$ was considered statistically significant.

\section{Results}

4.1. Screening of Active Components and Targets of XGHP. Xixin (XX) contains 192 components and Ganjiang (GJ) 148 components. All active ingredients met the screening rules, $\mathrm{OB} \geq 30 \%$ and $\mathrm{DL} \geq 0.18$. After screening, 12 core active compounds of XGHP were identified (Table 1), including 8 compounds in XX and 5 compounds in GJ. MOL002501 is a shared compound. A total of 428 potential targets were found for XGHP from TCMSP and DrugBank, and the UniProt database was used for standardization. Finally, 104 potential drug targets were obtained.

4.2. Targets of $X G H P$ against COPD. Using the GEO database microarray analysis (series: GSE130928, platforms: GPL570-55999), 700 differentially expressed genes were identified that were related to COPD (Table S1). Next, 2597 disease-related targets were identified by integrating the Human Gene Database, DisGeNET Database Disease Targets, and GEO databases after eliminating duplicates. By intersecting XGHP active component targets with COPD disease targets, 63 XGHP-COPD composite targets were obtained (Figure 2(a)). Subsequently, the "componentstargets" network of XGHP-COPD was established (Figure 2(b)). Kaempferol, $\beta$-sitosterol, and sesamin may be the central core components of XGHP-COPD.

4.3. PPI Network of Target Genes. The PPI network graph was obtained by importing 63 compound targets into STRING and by removing two disconnected points. There were 61 nodes and 442 edges, the average number of nodes was 14 , and the average local clustering coefficient was 0.579 . The TSV data was downloaded and imported into Cytoscape 3.7.2 to show the protein interaction network. Then, according to the three parameters DC, BC, and CC, the median target was selected as the key target for construction of the XGHP anti-COPD hub node. The screening criteria were $\mathrm{DC} \geqq 20, C C \geqq 0.6538$, and $\mathrm{BC} \geqq 0.8787$. The results included 18 hub nodes and 112 edges [22]. Finally, we adjusted the node properties in the network according to the degree value as follows: the larger the degree value of the target, the larger the area of the node (low values to dark colors) (Figure 2(c)).

4.4. Analyses of the Enrichment Pathway. In this study, we mainly found the life activities related to 18 hub targets in cellular composition, molecular function, and biological process. As the biological process was 760, XGHP treatment of COPD mainly involved the response to antibiotics, reactive oxygen species, and lipopolysaccharide, such as regulation of smooth muscle cell proliferation (GO: 0048660), regulation of DNA-binding transcription factor activity (GO:0051090), reactive oxygen species biosynthetic 
TABLE 1: A list of 12 active compounds.

\begin{tabular}{|c|c|c|c|c|c|}
\hline MOL & Compound & $\mathrm{OB}$ & $\mathrm{DL}$ & Degree & Herb \\
\hline MOL012140 & 4, 9-Dimethoxy-1-vinyl- $\beta$-carboline & 65.30 & 0.19 & 4 & $\mathrm{XX}$ \\
\hline MOL012141 & Caribine & 37.06 & 0.83 & 5 & $\mathrm{XX}$ \\
\hline MOL001460 & Cryptopine & 78.74 & 0.72 & 13 & $\mathrm{XX}$ \\
\hline MOL001558 & Sesamin & 56.55 & 0.83 & 7 & $\mathrm{XX}$ \\
\hline MOL002501 & $\begin{array}{l}{[(1 \mathrm{~S}) \text {-3-[(E)-but-2-enyl]-2-Methyl-4-oxo-1-cyclopent-2-enyl }](1 \mathrm{R}, 3 \mathrm{R})-3-[(\mathrm{E})-3 \text {-methoxy-2- }} \\
\text { methyl-3-oxoprop-1-enyl]-2, 2-dimethylcyclopropane-1-carboxylate }\end{array}$ & 62.52 & 0.31 & 1 & XX\&GJ \\
\hline MOL002962 & (3S)-7-Hydroxy-3-(2, 3, 4-trimethoxyphenyl)chroman-4-one & 48.23 & 0.33 & 12 & $\mathrm{XX}$ \\
\hline MOL000422 & Kaempferol & 41.88 & 0.24 & 39 & $\mathrm{XX}$ \\
\hline MOL009849 & ZINC05223929 & 31.57 & 0.83 & 2 & $\mathrm{XX}$ \\
\hline MOL002464 & 1-Monolinolein & 37.18 & 0.30 & 1 & GJ \\
\hline MOL002514 & Sexangularetin & 62.82 & 0.31 & 5 & GJ \\
\hline MOL000358 & Beta-sitosterol & 36.91 & 0.75 & 19 & GJ \\
\hline MOL000359 & Sitosterol & 36.91 & 0.75 & 1 & GJ \\
\hline
\end{tabular}

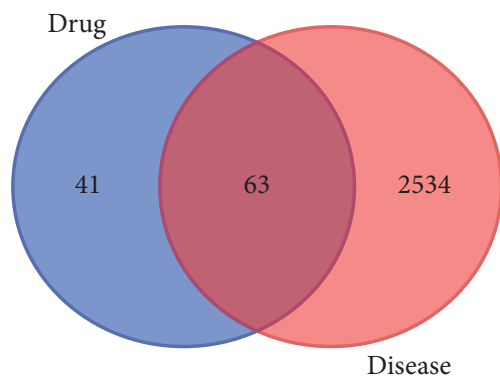

(a)

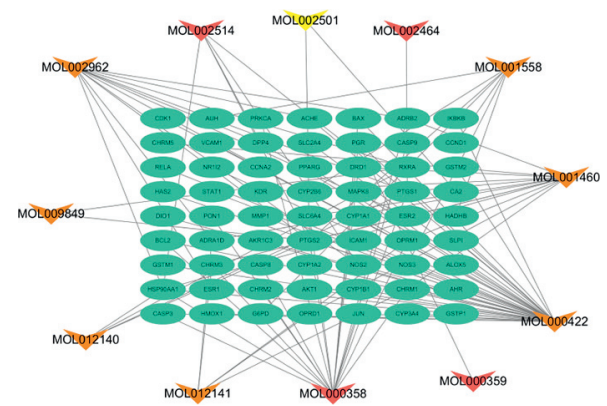

(b)
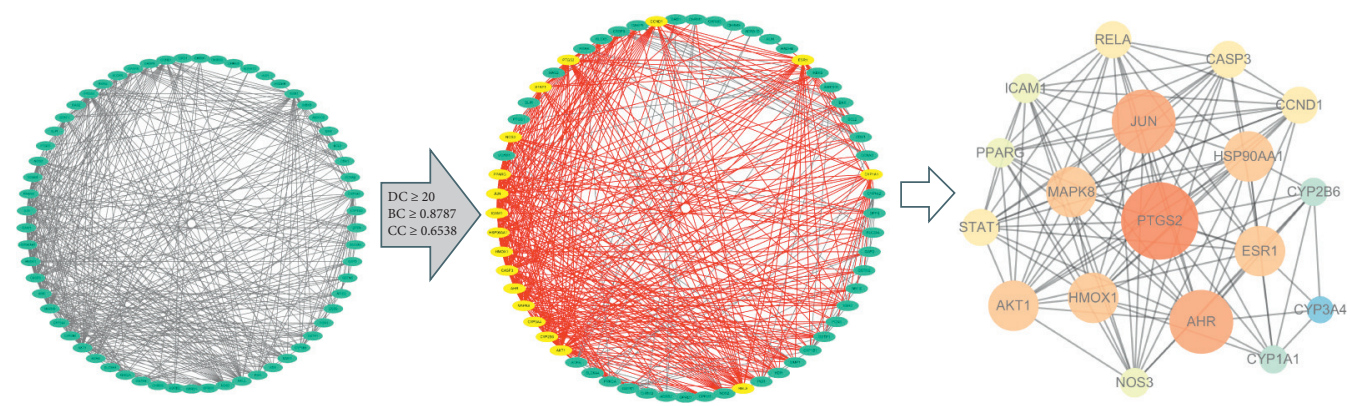

(c)

FIGURE 2: (a) Common targets between XGHP targets and COPD targets; (b) components-targets network. Orange nodes represent Xixin. Red nodes represent Ganjiang, and yellow nodes represent Xixin and Ganjiang, while green nodes represent targets of XGHP. (c) Topology filtering process of PPI network. 61 XGHP-COPD composite targets were screened by DC $\geq 20, \mathrm{BC} \geq 0.8787$, and CC $\geq 0.6538$, and finally, the PPI network of 18 hub nodes was obtained.

process (GO:1903409), and nitric oxide biosynthetic process (GO:0006809). The cellular composition was 8, which mainly involved membrane region (GO:0098589), transcription factor complex (GO:0005667), and nuclear chromatin (GO:0000790). The MF was 73. This mainly involved nuclear transcription, oxidoreductase activity, and heme binding, such as RNA polymerase II transcription factor binding (GO:0001085), nuclear receptor activity (GO: 0004879), and protein phosphatase binding (GO:0019903). Information on the top six enrichments in cellular composition, molecular function, and biological process were selected to create a bubble chart and bar chart. Moreover, the main signaling pathway of XGHP treatment for COPD was identified and analyzed by KEGG enrichment. KEGG pathway analysis returned 113 items, including the AGERAGE signaling pathway (hsa04933), TNF signaling pathway (hsa04668), and IL-17 signaling pathway (hsa04657) (Figure 3). The first 20 remarkable pathways were selected and presented in Table S2. Then, the network of the "Targetsbiological enrichment-pathways network" (Figure 4) was established.

\section{Validation}

5.1. Molecular Docking. In molecular docking, the less the binding energy between the ingredient and proteins is, the more likely the docking is to occur. In general, a compound with a binding fraction has a better binding activity with the 

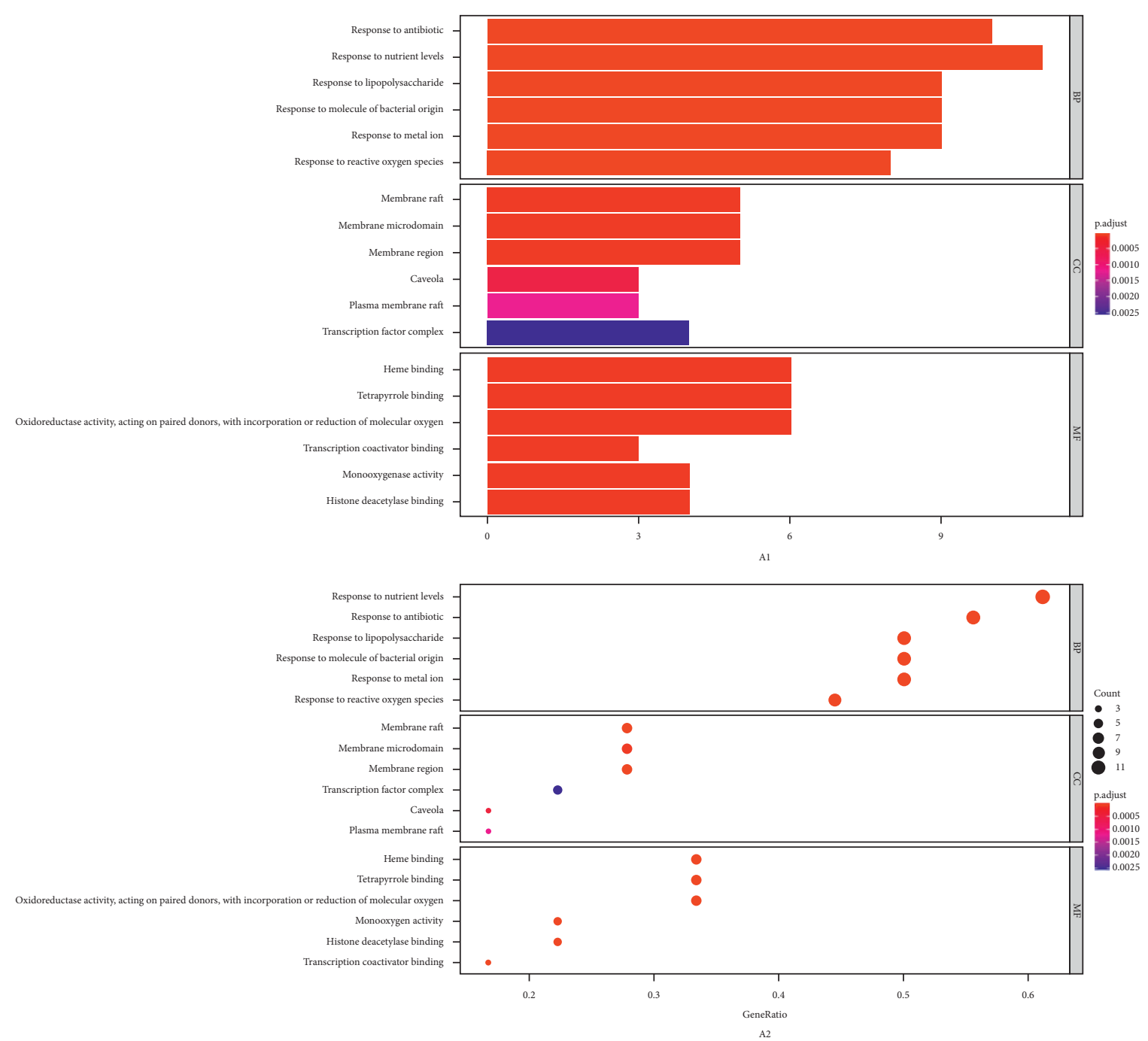

(a)
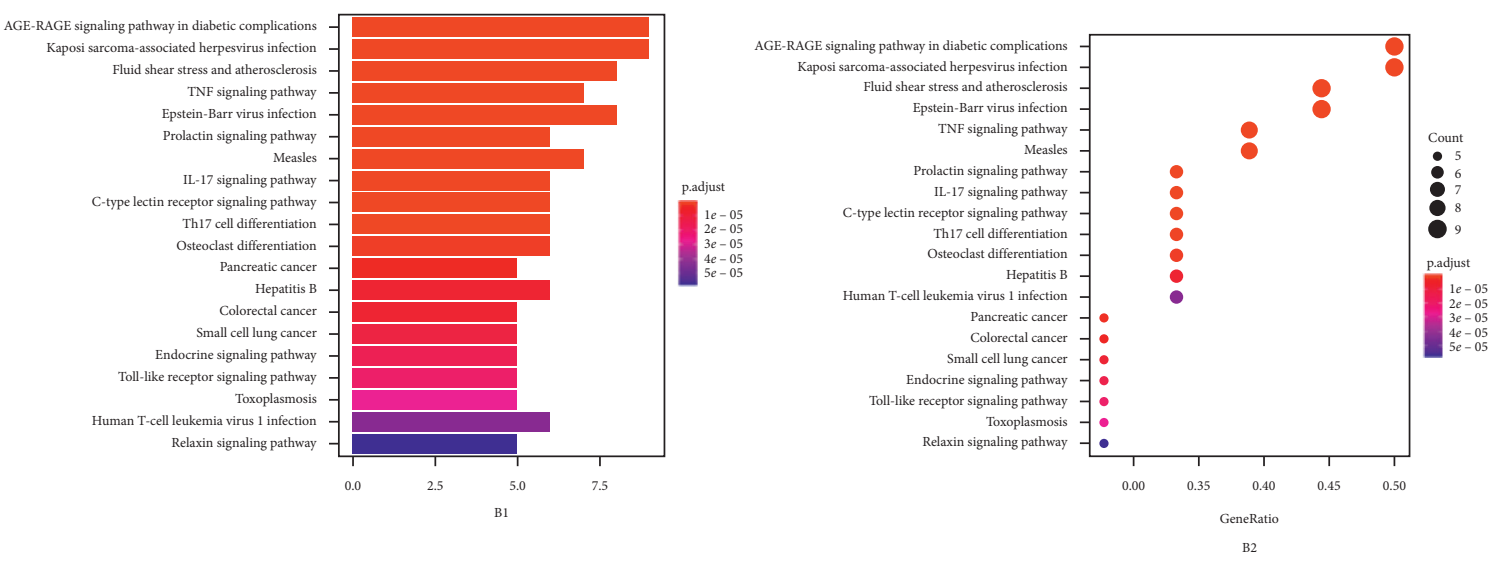

(b)

Figure 3: (a) A1 and A2: functional enrichment analysis based on GO database. (b) B1 and B2: pathway enrichment analysis based on KEGG database.

target. In this study, the 3 active components of degree, including kaempferol, sesamin, and $\beta$-sitosterol, were selected as candidate docking components. Moreover, PTGS2 and PPARG were chosen as the candidate targets, and aminophylline was selected as the positive drug for molecular docking. The results are presented in Figure 5. It can be concluded that kaempferol, sesamin, and $\beta$-sitosterol have a higher score than aminophylline for PTGS2 and 


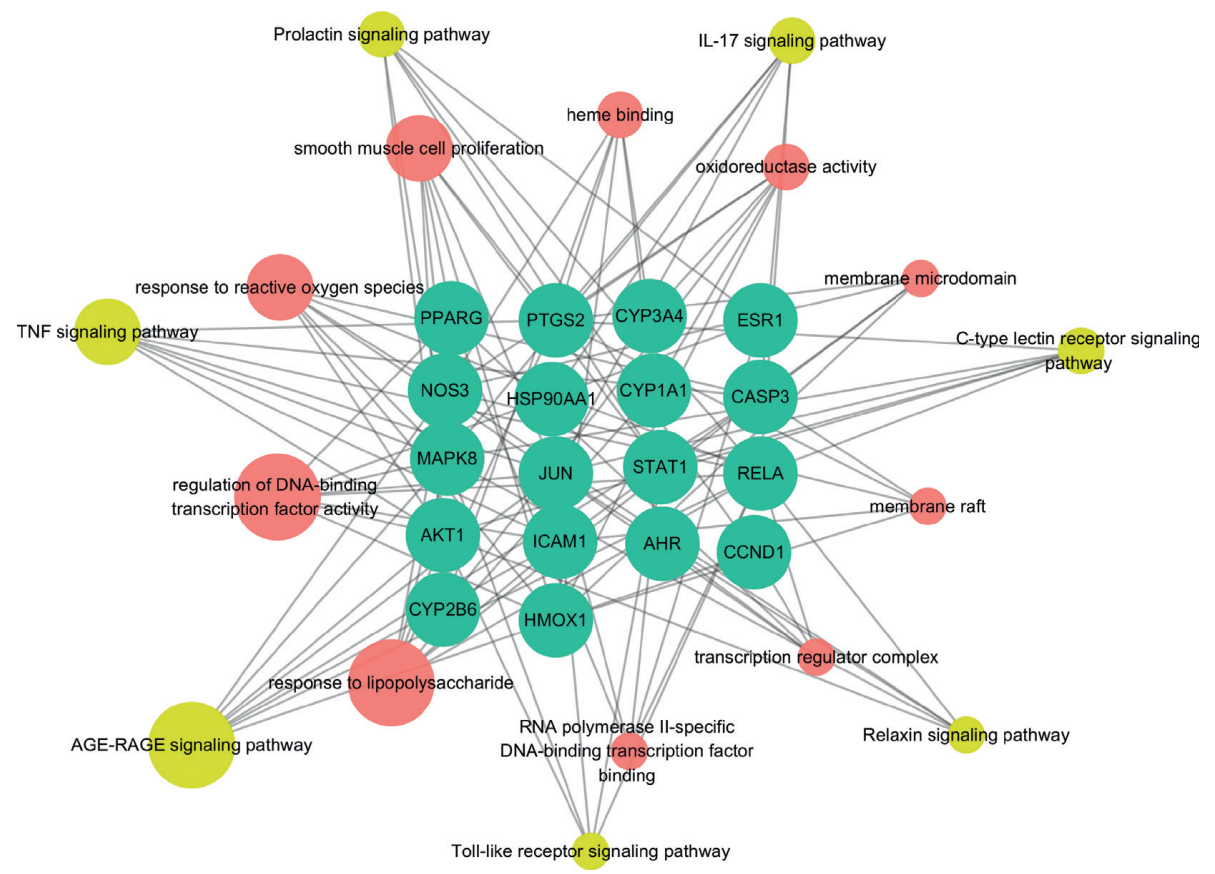

FIgURE 4: Networks for "Targets-biological enrichment-pathways.” The genes of XGHP-COPD showed up as green. The GO biological information showed up as pink. The related pathways showed up as yellow.

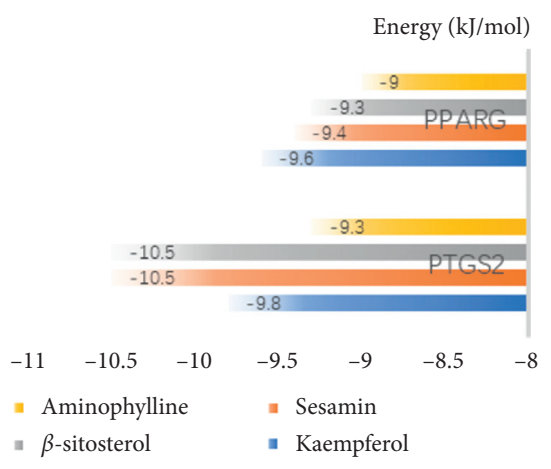

(a)

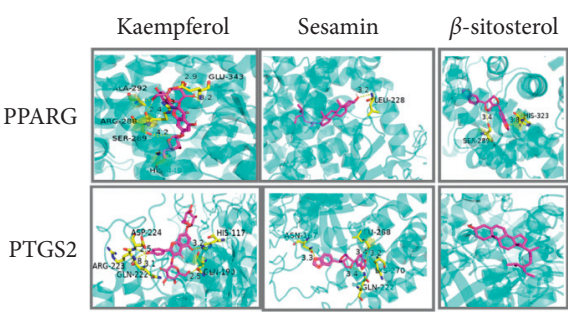

(b)

FIGURE 5: (a) Binding results of hub ingredients of XGHP and aminophylline with proteins. (b) Molecular docking model diagram of key pharmacodynamic substances-core targets.

PPARG, and it was suggested that the active components in XGHP closely bound to the predicted target, which may be the main basis for the treatment of COPD.

5.2. Western Blot Analysis. Based on network pharmacological analysis, PTGS2 and PPARG were selected for experimental verification. The results of Western blot analysis showed that that the PPARG protein content was significantly different between the normal group and the COPD group $(P<0.05)$. Treatment with XGHP or aminophylline showed that PPARG expression significantly increased in the $\mathrm{COPD}+\mathrm{XGHP}$ group or COPD + Ami group compared with the COPD group. The protein content of PTGS2 was markedly different between the COPD group and the normal group $(P<0.01)$. Furthermore, treatment with XGHP/aminophylline showed that PTGS2 expression significantly decreased in the COPD $+\mathrm{XGHP}$ group or COPD + Ami group compared with the COPD group $(P<0.05)$ (Figure 6).

\section{Discussion}

In the current study, network pharmacological was used to predict the substance basis and mechanism of action of XGHP on COPD. A total of 12 active ingredients and 104 corresponding core proteins were identified, and the active ingredients were closely linked to targets in the PPI network diagram. The KEGG signaling pathway also revealed that these targets were directly or indirectly involved in various inflammatory responses, including TNF signaling and IL-17 signaling. Moreover, molecular docking experiments revealed that the hub components in XGHP combined with 

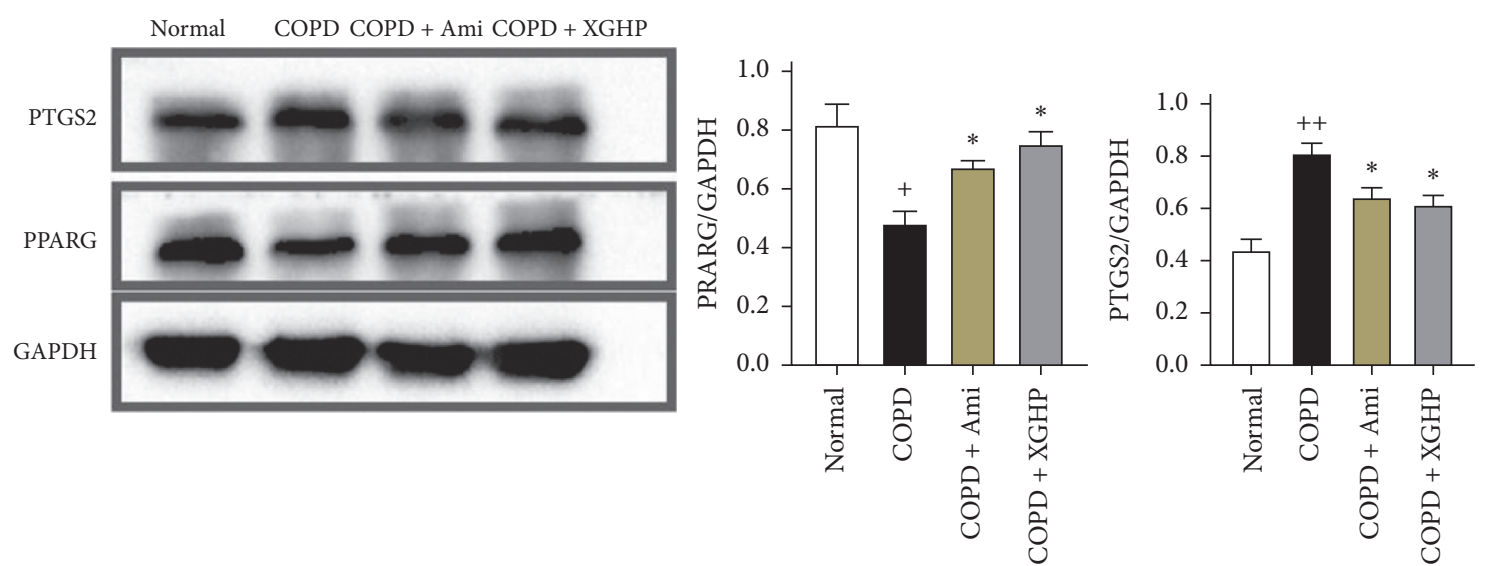

FIGURE 6: Effect of XGHP on PTGS2 and PPARG in lung in each group rats. ${ }^{++} \mathrm{P}<0.01$ versus the normal group; ${ }^{*} \mathrm{P}<0.05$ versus the COPD group. The value is expressed as mean \pm SEM.

the core targeted better than aminophylline. In the study, the expression of related inflammatory targets decreased or increased with the effect of XGHP, thereby indicating that Chinese medicine can effectively reduce airway inflammation.

Inflammation is a common clinical pathological process, which can occur in tissues and organs in various parts of the body. In general, inflammation is a type of antidisease response of the body, which is conducive to the recovery of balance of the body. However, under certain conditions, some beneficial factors in the inflammatory response can be transformed in the opposite direction and become harmful factors to the body. A growing body of evidence has shown that Chinese herbs can suppress inflammation. Mucus hypersecretion $(\mathrm{MH})$ in the airway is always accompanied by COPD, which is an independent risk factor affecting COPD disease change and prognosis [23]. Inhibition of several inflammatory pathways to reduce the inflammatory response can effectively reduce $\mathrm{MH}$ [24]. The efficacy and safety of XGHP in the treatment of COPD have been widely recognized. In this study, we focused on identifying the substance basis and pharmacological pathway of the role of XGHP.

In this study, kaempferol, $\beta$-sitosterol, and sesamin may be the important ingredients of XGHP in COPD treatment. Kaempferol, one of the essential ingredients, was found in 12 active ingredients of XGHP with 39 targets. In many studies, it has been shown that kaempferol can reduce various types of inflammation induced by the lipopolysaccharide (LPS), such as colitis, neuroinflammation, and rheumatoid arthritis [25-27]. LPS-induced inflammation is often used as one of the methods to establish COPD models. The mechanism of action of kaempferol is to inhibit the nuclear translocation of signal transducer and activator of transcription $[28,29]$, thereby inhibiting the expression of PTGS2 and activating the NF- $\kappa \mathrm{B}$ signaling pathway $[30,31] . \beta$-sitosterol, a sterol commonly found in herbs, was used to treat pathological changes in lung tissue and prevent airway inflammation [32] and also showed anti-inflammatory effects in macrophages, microglia, and joint tissues [33-35]. Moreover, $\beta$-sitosterol could reduce the LPS-induced expression of PTGS2 and exert anti-inflammatory and analgesic effects [36]. Kaempferol, $\beta$-sitosterol, and sesamin can enhance insulin resistance, protect myocardial cells from injury, and prevent hyperlipidemia by increasing the expression of PPARG $[37,38]$. Sesamin also reduced the overexpression of PTGS2 by regulating the JNK and p38 MAP kinase pathways [39]. In vitro experiments showed that sesamin could significantly inhibit the expression of PTGS2 in a dose-dependent manner $[40,41]$. In the literature, these three active ingredients have shown significant anti-inflammatory effects, which indicated that anti-inflammatory effects are an important drug basis of XGHP and an essential link in the treatment of COPD.

A significant decrease in the number of various inflammatory cells in the administration group was found to be related to the inhibition of PTGS2 expression [42]. Wang et al. demonstrated that the inhibition of PTGS2 could regulate the expression of aquaporin-1 and alleviate lung injury [43]. Furthermore, PTGS2 was directly enriched in related inflammation pathways, such as the TNF signaling pathway and the IL-17 signaling pathway, and in small cell lung cancer. Moreover, PTGS2 and PPARG were directly connected in the PPI network, thus suggesting that XGHP exerts an anti-inflammatory effect through a multitarget and multipathway combination rather than single pathway. PPARG, a subtype of PPARs, is a nuclear receptor family member. PPARG and its ligands play a vital role in lipid and glucose metabolism. Cho et al. demonstrated that rosiglitazone (PPARG agonist) could protect lung tissues by inhibiting NOx/ROS/C-SRC/PYK2/ Akt-dependent activation of NRF2, either by inducing HO-1 expression or by inhibiting NF- $\kappa$ B expression, thereby further inhibiting activation of TLR2/NLRP3 inflammasomes [44]. In addition, a variety of in vitro experiments have demonstrated that PPARG can regulate mucinous proteins and inflammatory cytokines in lung tissue, thereby exaggerating the expression of airway hyperresponsiveness, inflammation, and cytokines $[45,46]$. Taken together, these results indicated that PPARG can improve pulmonary inflammation through various signaling pathways. 


\section{Conclusion}

In conclusion, we predicted and verified that XGHP is beneficial to restrain the expression of PTGS2 in lung tissue and promote the expression of PPARG through various inflammation-related pathways, thus treating COPD and restoring lung function. Although the results reveal that XGHP may influence COPD through inflammatory responses and LPS and partially explain the relevant targets of anti-COPD, we still need to further verify the relevant pathways involved and explore the pharmacological mechanism of COPD treatment.

\section{Abbreviations}

$\begin{array}{ll}\text { COPD: } & \text { Chronic obstructive pulmonary disease } \\ \text { XGHP: } & \text { Xixin-Ganjiang herb pair } \\ \text { XX: } & \text { Xixin (Asarum) } \\ \text { GJ: } & \text { Ganjiang (Zingiberis Rhizoma) } \\ \text { GEO: } & \text { Gene Expression Omnibus } \\ \text { PPI: } & \text { Protein-protein interaction } \\ \text { GO: } & \text { Gene ontology } \\ \text { KEGG: } & \text { Kyoto Encyclopedia of Genes and Genomes } \\ \text { TCM: } & \text { Traditional Chinese Medicine } \\ \text { MUC5AC: } & \text { Mucin 5AC } \\ \text { OB: } & \text { Oral bioavailability } \\ \text { DL: } & \text { Drug-likeness } \\ \text { FC: } & \text { Fold change } \\ \text { DC: } & \text { Degree of centricity } \\ \text { CC: } & \text { Closeness centrality } \\ \text { BC: } & \text { Betweenness centrality } \\ \text { SD: } & \text { Sprague Dawley } \\ \text { Ami: } & \text { Aminophylline } \\ \text { LPS: } & \text { Lipopolysaccharide } \\ \text { PDB: } & \text { Protein Data Bank } \\ \text { PMSF: } & \text { Phenyl methyl sulfonyl fluoride } \\ \text { SDS-PAGE: } & \text { Polyacrylamide gel electrophoresis } \\ \text { PVDF: } & \text { Polyvinylidene fluoride } \\ \text { TBST: } & \text { Tris buffer solution Tween } \\ \text { WB: } & \text { Western blot } \\ \text { HO-1: } & \text { Heme oxygenase. } \\ & \end{array}$

\section{Data Availability}

The data used to support the findings of this study are available from the corresponding author upon request.

\section{Conflicts of Interest}

The authors state that they have no conflicts of interest in this work.

\section{Authors' Contributions}

Qiong Wang, Bailu Duan, and Zhenxiang Zhou conceived and designed the study. Deshun Li, Jingjing Li, Jiajia Wu, and Lintao Han analyzed the data. Ping Huang and Tao Huang wrote the manuscript. Ping Huang, Bailu Duan, and Yan Ye carried out the experiment. All authors read and approved the final version of the manuscript. Ping Huang and Tao Huang contributed equally to this work.

\section{Acknowledgments}

This study was funded by the National Natural Science Foundation of China (Grant no. 81903815) and Science and Technology Research Project of Hubei Provincial Department of Education (Grant no. B2019100).

\section{Supplementary Materials}

Table S1: differentially expressed genes in GEO chip. Table S2: top 20 KEGG pathway analysis. (Supplementary Materials)

\section{References}

[1] B. S. A. Silva, D. Ramos, G. N. Bertolini et al., "Resistance exercise training improves mucociliary clearance in subjects with COPD: a randomized clinical trial," Pulmonology, vol. 25, no. 6, pp. 340-347, 2019.

[2] Y. W. Kim, C. H. Lee, H. G. Hwang et al., "Resting hyperinflation and emphysema on the clinical course of COPD," Scientific Reports, vol. 9, no. 1, p. 3764, 2019.

[3] E. Diaz-Guzman and D. M. Mannino, "Epidemiology and prevalence of chronic obstructive pulmonary disease," Clinics in Chest Medicine, vol. 35, no. 1, pp. 7-16, 2014.

[4] V. K. Vijayan, "Chronic obstructive pulmonary disease," Indian Journal of Medical Research, vol. 137, no. 2, pp. 251269, 2013.

[5] K. Takeuchi, K. Matsumoto, M. Furuta et al., "Periodontitis is associated with chronic obstructive pulmonary disease," Journal of Dental Research, vol. 98, no. 5, pp. 534-540, 2019.

[6] Y. Wang, J. Xu, Y. Meng, I. M. Adcock, and X. Yao, "Role of inflammatory cells in airway remodeling in COPD," International Journal of Chronic Obstructive Pulmonary Disease, vol. 13, pp. 3341-3348, 2018.

[7] E. Bagdonas, J. Raudoniute, I. Bruzauskaite et al., "Novel aspects of pathogenesis and regeneration mechanisms in COPD," International Journal of Chronic Obstructive Pulmonary Disease, vol. 10, pp. 995-1013, 2015.

[8] Q. Song, D. Christiani, W. Xiaorong, and J. Ren, "The global contribution of outdoor air pollution to the incidence, prevalence, mortality and hospital admission for chronic obstructive pulmonary disease: a systematic review and metaanalysis," International Journal of Environmental Research and Public Health, vol. 11, no. 11, pp. 11822-11832, 2014.

[9] S. Wang, Y. Hu, W. Tan et al., "Compatibility art of traditional Chinese medicine: from the perspective of herb pairs," Journal of Ethnopharmacology, vol. 143, no. 2, pp. 412-423, 2012.

[10] N. Chen and Q. Yang, "Effect of Xiaoqinglong decoction on MUC5AC and QOL of patients with airway mucus hypersecretion," Information on Traditional Chinese Medicine, vol. 37, no. 5, pp. 106-109, 2020.

[11] Y. H. Ma, Y. Li, N. Guo et al., "Linggan Wuwei Jiangxin decoction assisting treatment of lung diseases," Acta Chinese Medicine, vol. 35, no. 10, pp. 2128-2132, 2020.

[12] R. K. Li, Y. Li, Y. R. An et al., "Effects of Linggan Wuwei Jiangxin Decoction on mrna and protein expressions of P-CREB and MUC5ACmRNA in asthmatic rats," Lishizhen Medicine and Materia Medica Research, vol. 27, no. 9, pp. 2126-2129, 2016. 
[13] M. Kibble, N. Saarinen, J. Tang, K. Wennerberg, S. Mäkelä, and T. Aittokallio, "Network pharmacology applications to map the unexplored target space and therapeutic potential of natural products," Natural Product Reports, vol. 32, no. 8, pp. 1249-1266, 2015.

[14] R. Zhang, X. Zhu, H. Bai et al., "Network pharmacology databases for traditional Chinese medicine: review and assessment," Frontiers in Pharmacology, vol. 10, p. 123, 2019.

[15] B. Duan, L. Han, S. Ming et al., "Fuling-guizhi herb pair in coronary heart disease: integrating network pharmacology and in vivo pharmacological evaluation," Evidence-Based Complementary and Alternative Medicine, vol. 2020, Article ID 1489036, 10 pages, 2020.

[16] J. Ru, P. Li, J. Wang et al., "TCMSP: a database of systems pharmacology for drug discovery from herbal medicines," Journal of Cheminformatics, vol. 6, p. 13, 2014.

[17] X. Xu, W. Zhang, C. Huang et al., "A novel chemometric method for the prediction of human oral bioavailability," International Journal of Molecular Sciences, vol. 13, no. 6, pp. 6964-6982, 2012.

[18] C. Von Mering, L. J. Jensen, B. Snel et al., "STRING: known and predicted protein-protein associations, integrated and transferred across organisms," Nucleic Acids Research, vol. 33, no. Database issue, pp. D433-D437, 2005.

[19] S. Gu, Y. Xue, Y. Gao et al., "Mechanisms of indigo naturalis on treating ulcerative colitis explored by GEO gene chips combined with network pharmacology and molecular docking," Scientific Reports, vol. 10, no. 1, p. 15204, 2020.

[20] G. Yu, L.-G. Wang, Y. Han, and Q.-Y. He, "clusterProfiler: an $\mathrm{R}$ Package for comparing biological themes among gene clusters," OMICS: A Journal of Integrative Biology, vol. 16, no. 5, pp. 284-287, 2012.

[21] X. G. Zhong, Y. H. Li, X. Jia et al., "Influence of therapy of dredging large intestine on airway mucus hypersecretion in COPD rats," Journal of Beijing University of Traditional Chinese Medicine, vol. 33, no. 12, pp. 809-812+871, 2010.

[22] Y. Wan, L. Xu, Z. Liu et al., "Utilising network pharmacology to explore the underlying mechanism of Wumei Pill in treating pancreatic neoplasms," BMC Complementary Medicine and Therapies, vol. 19, no. 1, p. 158, 2019.

[23] Y. C. Shen and F. Q. Wen, "Interpretation of Chinese expert consensus on the management of airway mucus hypersecretion in chronic airway inflammatory diseases," Chinese Journal of Practical Internal Medicine, vol. 36, no. 2, pp. p131-132+136, 2016.

[24] F. Yin, "Listeria hemolysin promotes inflammatory response and MUC5AC expression in respiratory epithelial cells by activating PI3K/Akt signaling pathway," Chinese Journal of Immunology, vol. 35, no. 3, pp. p282-286, 2019.

[25] Y. L. Yang, X. Cheng, W. H. Li et al., "Kaempferol attenuates LPS-induced striatum injury in mice involving anti-neuroinflammation, maintaining BBB integrity, and down-regulating the HMGB1/TLR4 pathway," International Journal of Molecular Sciences, vol. 20, no. 3, 2019.

[26] M.-Y. Park, G. E. Ji, and M.-K. Sung, "Dietary kaempferol suppresses inflammation of dextran sulfate sodium-induced colitis in mice," Digestive Diseases and Sciences, vol. 57, no. 2, pp. 355-363, 2012.

[27] H.-Y. Yoon, E.-G. Lee, H. Lee et al., "Kaempferol inhibits IL-1 $\beta$ induced proliferation of rheumatoid arthritis synovial fibroblasts and the production of COX-2, PGE2 and MMPs," International Journal of Molecular Medicine, vol. 32, no. 4, pp. 971-977, 2013.

[28] S.-B. Lee, J.-S. Shin, H.-S. Han, H.-H. Lee, J. C. Park, and K.-T. Lee, "Kaempferol 7-O- $\beta$-D-glucoside isolated from the leaves of Cudrania tricuspidata inhibits LPS-induced expression of pro-inflammatory mediators through inactivation of NF- $\kappa$ B, AP-1, and JAK-STAT in RAW 264.7 macrophages," Chemico-Biological Interactions, vol. 284, pp. 101-111, 2018.

[29] Q. Yu, K. Zeng, X. Ma et al., "Resokaempferol-mediated anti-inflammatory effects on activated macrophages via the inhibition of JAK2/STAT3, NF- $\kappa$ B and JNK/p38 MAPK signaling pathways," International Immunopharmacology, vol. 38, pp. 104-114, 2016.

[30] D. R. Kang, S. A. Belal, H. S. Choe, D. K. Shin, and K. S. Shim, "Effect of kaempferol on cyclooxygenase 2 (Cox2) and cytosolic phospholipase A2 (cPLA2) protein expression in BALB/c mice," Iranian Journal of Allergy, Asthma and Immunology, vol. 17, no. 5, pp. 428-435, 2018.

[31] W. Alam, H. Khan, M. A. Shah et al., "Kaempferol as a dietary anti-inflammatory agent: current therapeutic standing," Molecules, vol. 25, no. 18, 2020.

[32] S. G. Mahajan and A. A. Mehta, "Suppression of ovalbumininduced Th2-driven airway inflammation by $\beta$-sitosterol in a Guinea pig model of asthma," European Journal of Pharmacology, vol. 650, no. 1, pp. 458-464, 2011.

[33] L. Yuan, F. Zhang, M. Shen et al., "Phytosterols suppress phagocytosis and inhibit inflammatory mediators via ERK pathway on LPS-triggered inflammatory responses in RAW264.7 macrophages and the correlation with their structure," Foods, vol. 8, no. 11, 2019.

[34] F. Zhang, Z. Liu, X. He, Z. Li, B. Shi, and F. Cai, " $\beta$-Sitosterolloaded solid lipid nanoparticles ameliorate complete Freund's adjuvant-induced arthritis in rats: involvement of NF-KB and HO-1/Nrf-2 pathway," Drug Delivery, vol. 27, no. 1, pp. 1329-1341, 2020.

[35] Y. Sun, L. Gao, W. Hou et al., "Beta-sitosterol alleviates inflammatory response via inhibiting the activation of ERK/p38 and NF-kappaB pathways in LPS-exposed BV2 cells," BioMed Research International, vol. 2020, Article ID 7532306, 10 pages, 2020.

[36] Q. Yang, D. Yu, and Y. Zhang, "Beta-sitosterol attenuates the intracranial aneurysm growth by suppressing TNF-alphamediated mechanism," Pharmacology, vol. 104, no. 5-6, pp. 303-311, 2019.

[37] F. Lin, L. Xu, M. Huang et al., "Beta-sitosterol protects against myocardial ischemia/reperfusion injury via targeting PPARgamma/NF-kappaB signalling," Evidence-Based Complementary and Alternative Medicine, vol. 2020, Article ID 2679409, 9 pages, 2020.

[38] S. Ramalingam, M. Packirisamy, M. Karuppiah et al., "Effect of $\beta$-sitosterol on glucose homeostasis by sensitization of insulin resistance via enhanced protein expression of $\mathrm{PPR} \gamma$ and glucose transporter 4 in high fat diet and streptozotocininduced diabetic rats," Cytotechnology, vol. 72, no. 3, pp. 357-366, 2020.

[39] T. Y. Lin, P. Y. Wu, C. W. Hou et al., "Protective effects of sesamin against UVB-induced skin inflammation and photodamage in vitro and in vivo," Biomolecules, vol. 9, no. 9, 2019.

[40] K. Li, Y. Li, B. Xu, L. Mao, and J. Zhao, "Sesamin inhibits lipopolysaccharide-induced inflammation and extracellular matrix catabolism in rat intervertebral disc," Connective Tissue Research, vol. 57, no. 5, pp. 347-359, 2016.

[41] A.-M. Rousta, S.-M.-S. Mirahmadi, A. Shahmohammadi et al., "Protective effect of sesamin in lipopolysaccharide-induced mouse model of acute kidney injury via attenuation of oxidative stress, inflammation, and apoptosis," 
Immunopharmacology and Immunotoxicology, vol. 40, no. 5, pp. 423-429, 2018.

[42] J. Yuan, R. Liu, Y. Ma, Z. Zhang, and Z. Xie, "Curcumin attenuates airway inflammation and airway remolding by inhibiting NF- $\kappa \mathrm{B}$ signaling and COX-2 in cigarette smokeinduced COPD mice," Inflammation, vol. 41, no. 5, pp. 1804-1814, 2018.

[43] L. Wang, Y. Shan, Y. Ye et al., "COX-2 inhibition attenuates lung injury induced by skeletal muscle ischemia reperfusion in rats," International Immunopharmacology, vol. 31, pp. 116-122, 2016.

[44] R.-L. Cho, C.-C. Yang, H.-C. Tseng, L.-D. Hsiao, C.-C. Lin, and C.-M. Yang, "Haem oxygenase-1 up-regulation by rosiglitazone via ROS-dependent Nrf2-antioxidant response elements axis or PPAR $\gamma$ attenuates LPS-mediated lung inflammation," British Journal of Pharmacology, vol. 175, no. 20, pp. 3928-3946, 2018.

[45] S. P. Lakshmi, A. T. Reddy, A. Banno, and R. C. Reddy, "Airway epithelial cell peroxisome proliferator-activated receptor $\gamma$ regulates inflammation and mucin expression in allergic airway disease," The Journal of Immunology, vol. 201, no. 6, pp. 1775-1783, 2018.

[46] S. Huang, B. Zhu, I. S. Cheon et al., "PPAR-gamma in macrophages limits pulmonary inflammation and promotes host recovery following respiratory viral infection," Journal of Virology, vol. 93, no. 9, 2019. 